\title{
Roland Barthes : la résurrection du stéréotype
}

\author{
Daniele CARLUCCIO \\ Université de Genève
}

\begin{abstract}
La Doxa est un mauvais objet parce que c'est une répétition morte, qui ne vient du corps de personne - sinon peut-être, précisément, de celui des Morts ", dit Roland Barthes dans un fragment de son autobiographie. La reformulation suggère qu'il y a bien un " corps ", sous la Doxa. Le présent article est consacré à cette hantise barthésienne de la chose doxique, telle qu'elle se manifeste dans les Mythologies, à propos des mythes, ces « cadavres parlants ». Le commentaire prend pour objet particulier le texte sur le catch, lu à la lumière de l'essai sur Michelet et du thème, cher à l'historien, de la résurrection. Il s'attache à montrer que le mythe christique, comme le rapprochement avec la tragédie, transfigure le mythe prosaïque du catch, dans la vision et dans l'écriture de Barthes.
\end{abstract}

Keywords : Barthes, stéréotype, mythe, traumatisme, catch

« La Doxa est un mauvais objet parce que c'est une répétition morte, qui ne vient du corps de personne - sinon peut-être, précisément, de celui des Morts $»^{1}$, dit Roland Barthes dans un fragment de son autobiographie. Énoncé retors : la reformulation suggère qu'il y a bien quelqu'un, un "corps ", sous la Doxa. Quant à la majuscule, typique de l'écrivain, sa valeur oscille entre conceptualité philosophique (" la Doxa )) et gravité mélancolique ( les Morts »). C'est à cette hantise barthésienne de la chose doxique, stéréotype ou mythe, que ces pages seront consacrées. Dans la réflexion de Barthes sur la littérature et la culture, la question du lieu commun revient constamment et ses commentateurs n'ont pas manqué de s'y arrêter. Ruth Amossy accorde une place de choix à Barthes dans sa Sémiologie des stéréotypes $^{2}$. Philippe Jousset et, tout récemment, Lise Forment ont versé encore des pièces à ce dossier, l'un explorant, chez l'auteur des Mythologies et de $L a$ Chambre claire, l'association toute moderne, fondée sur l'homonymie, entre cliché de langage et cliché photographique ${ }^{3}$; l'autre analysant sa critique de la rhétorique et son rapport, en partie manqué, à la dissidence prémoderne ${ }^{4}$.

I Roland Barthes, Roland Barthes par Roland Barthes, in Euvres complètes, 5 vol., éd. Éric Marty, Paris, Seuil, 2002, t. V, p. 649.

2 Ruth Amossy, Les Idées reçues. Sémiologie du stéréotype, Paris, Nathan, 199I, pp. 77-96. Voir également Ruth Amossy et Anne Herschberg Pierrot, Stéréotypes et clichés, Paris, Nathan, 1997, pp. 62-64.

3 Philippe Jousset, "Morales du cliché. Le retour des évidences », La Licorne, 59, décembre 200I, pp. 297-308.

4 Lise Forment, "Classicisme et rhétorique chez Roland Barthes : que faire des lieux communs du XVII e siècle dans la "modernité" ? ", in Politique des lieux communs, éds. Lise Forment, 
Je signale à dessein ces deux contributions car elles manifestent toutes deux que cette question du lieu commun, chez Barthes, comme chez Jean Paulhan, ne cesse de renvoyer indissociablement à la situation du moderne, dans la modernité et vis-à-vis du classicisme. Le « lieu commun », ou «topos », le " cliché », le «stéréotype » : plusieurs termes pour un même objet. On peut y observer des nuances, proposer à partir de là une catégorisation, comme l'a fait Pascal Durand'. Mais pour ma part, je m'arrêterai moins au sens particulier de ces termes qu'à leur origine, permettant de retracer cette ligne de partage traditionnelle et schématique entre classicisme et modernité. Classicisme du topos, du lieu d'autant plus commun qu'on l'imagine pratiquement toujours habité, comme lorsqu'on regarde une carte et que l'on y observe des toponymes, derrière lesquels on voit se dessiner des constructions, des rues, des places, où l'on devine des personnes qui y évoluent. Le lieu commun, donc, au sens de cette sagesse communautaire sur laquelle Aristote a fondé sa Rhétorique et, au-delà, une vision habitable et habitée du monde ${ }^{6}$. Modernité, à l'autre bord, du « cliché » et du « stéréotype », qui renvoient au développement technique de l'imprimerie (l'utilisation, à partir du début du XIX siècle, de planches à caractères fixes) et, allégoriquement, à une standardisation redoutée de la production culturelle - où le lieu commun devient trop commun, c'est-à-dire qu'il ne l'est plus vraiment, qu'il est déserté, inhabité, voire inhabitable. Autrement dit, qu'il semble se répéter en l'absence de tout sujet.

Il sera donc question de la modernité du stéréotype, de la modernité comme âge de la répétition, selon la vision bien connue de Walter Benjamin 7 . D'une telle répétition, toujours traumatique, je trouve une image terrible et poétique dans l'agonie de ce chimiste hongrois appelé Sómogyi, telle que la relate Primo Levi à la fin de Si c'est un homme. Alors que le camp d'Auschwitz - toponyme hanté comme nul autre - a été abandonné par les nazis en fuite et les survivants livrés à eux-mêmes, Sómogyi, pris d'une violente fièvre, fait don de sa dernière ration de pain et s'enferme dans le mutisme. « Mais le soir, et toute la nuit, et pendant deux longs jours, le délire eut raison de son silence », raconte Levi avant de poursuivre :

Livré à un ultime et interminable rêve de soumission et d'esclavage, il se

Tiphaine Pocquet et Léo Stambul, Rennes, Presses universitaires de Rennes, 2016, pp. 185-203. 5 Voir Pascal Durand, «Lieu commun et communication. Concepts et application critique ", in Médias et censure. Figures de l'orthodoxie, éd. Pascal Durand, Liège, Éditions de l'Université de Liège, 2004, pp. 83-108.

6 Voir Anne Cauquelin, L’Art du lieu commun. Du bon usage de la doxa, Paris, Seuil, 1999, pp.3644 .

7 Dans «L'Euvre d'art à l'ère de sa reproductibilité technique » [1955] et «Sur quelques thèmes baudelairiens » [1939] (Walter Benjamin, Euvres, 3 vol., trad. Maurice de Gandillac, Rainer Rochlitz et Pierre Rusch, Paris, Gallimard, 2000, t. III, pp. 269-3I6 et 329-390). 
mit à murmurer « Jawohl » chaque fois qu'il respirait, au rythme continu et régulier d'une machine ; «Jawohl » à chaque fois que sa pauvre cage thoracique s'abaissait, « Jawohl » des milliers de fois, à nous faire venir l'envie de le secouer, de l'étouffer, ou au moins de l'obliger à dire autre chose ${ }^{8}$.

L'image peut paraître excessivement noire, elle nous rapproche pourtant de Barthes, qui évoque une scène que l'on pourrait qualifier de semblable, s'il était permis de faire abstraction du poids historique du témoignage de Levi'. Il s'agit, dans Le Plaisir du texte, de l'agonie interminable de M. Valdemar, le personnage du conte d'Edgar Poe :

La nausée arrive dès que la liaison de deux mots importants va de soi. Et dès qu'une chose va de soi, je la déserte : c'est la jouissance. Agacement futile? Dans la nouvelle d'Edgar Poe, M. Valdemar, le mourant magnétisé, survit, cataleptique, par la répétition des questions qui lui sont adressées (« M. Valdemar, dormez-vous ?») ; mais cette survie est intenable : la fausse mort, la mort atroce, c'est ce qui n'est pas un terme, c'est l'interminable (« Pour l'amour de Dieu ! - Vite ! - Vite ! - faites-moi dormir, - ou bien vite ! éveillez-moi vite! - Je vous dis que je suis mort !»). Le stéréotype, c'est cette impossibilité nauséeuse de mourir ${ }^{10}$.

Répétition, donc, et non remémoration. Répétition, pour Sómogyi, de l'interjection qui dit son asservissement au bourreau nazi, jusqu'à la mort et au-delà. Répétition, dans le cas de Valdemar, des questions qui lui sont adressées et des réponses qu'il donne depuis l'« impossible » lieu ou état où le maintient l'hypnose. Sauf que ce que répète Sómogyi est la trace d'un asservissement réel et vécu, et non d'un asservissement morbide au langage lui-même, comme c'est le cas dans la lecture que Barthes fait du Cas de M. Valdemar, lecture tributaire de celles de Lacan et de Derrida dans les années précédentes, qui l'une comme l'autre voient dans le Mot le meurtre de la Chose, avec en toile de fond les considérations de Blanchot sur Mallarmé et la négativité littéraire ${ }^{\mathrm{II}}$. C'est le langage lui-même qui est négativité, et de

8 Primo Levi, Si c'est un homme, trad. Martine Schruoffeneger, Paris, Robert Laffont, 1996, p. 229.

9 Je note que Barthes, dans les Fragments d'un discours amoureux, a lui-même eu la hardiesse de rapprocher le traumatisme du concentrationnaire de la souffrance d'amour : « L'une des injures les plus inimaginables de l'Histoire peut-elle se retrouver dans un incident futile, enfantin, sophistiqué, obscur, advenu à un sujet confortable, qui est seulement la proie de son Imaginaire ? Ces deux situations ont néanmoins ceci de commun : elles sont, à la lettre, paniques " (Barthes, Euvres complètes, op. cit., t. V, p. 80).

Io Id., Le Plaisir du texte, in Euvres complètes, op. cit., t. IV, pp. 245-246.

II Voir Maurice Blanchot, «Le Mythe de Mallarmé » et «La Littérature et le Droit à la mort », in La Part du feu, Paris, Gallimard, 1949, pp.35-48 et 29I-33I ; Jacques Derrida, La Voix et le Phénomène, Paris, Presses universitaires de France, 1967, p. I08; et Jacques Lacan, Le Séminaire, 1. II : "Le Moi dans la théorie de Freud et dans la technique de la psychanalyse », éd. Jacques-Alain 
cette expérience du négatif les traumatismes individuels ou historiques ne sont jamais que l'après-coup. Rapprocher ces deux fragments du Plaisir du texte et de Si c'est un homme, c'est donc prendre le parti d'un Barthes tragiquement moderne $\mathrm{I}^{\mathrm{I}}$, dans son rapport au stéréotype, et au mythe.

\section{Le mythe n'est pas une parole}

Je centrerai mon propos sur les Mythologies, qui a longtemps été l'œuvre la plus lue et la plus connue de Barthes, avant d'être détrônée d'abord par les Fragments d'un discours amoureux, à la fin des années 70, puis de nos jours par La Chambre claire, deux œuvres dont la critique a montré qu'elles peuvent être considérées comme l'autre livre du mythologue, où à la critique succède le retour au réel ${ }^{13}$. Les Mythologies sont un recueil de textes parus au milieu des années 50 prenant tous pour objet l'actualité médiatique, suivi d'un texte plus long qui en constitue la théorisation a posteriori, qui donc s'applique à définir ce que Barthes entend par «mythe » et par «mythologie ». Dans cette postface, Barthes commence par expliquer, en s'appuyant sur l'étymon, muthos, que «le mythe est une parole ${ }^{14}$. Cette définition lapidaire est en quelque sorte une facilité, encouragée par l'étymologie, puisqu'il apparaît rapidement que le mythe n'est pas, fondamentalement, une parole, ni un récit, comme le veut le sens commun (ou lévi-straussien) du mot, mais, bien davantage, une image, un fragment visuel de la réalité, capturé et parasité par une parole. Jacqueline Guittard, qui a procuré une édition illustrée des Mythologies, a bien montré que l'image, au sens concret du cliché trouvé dans la presse populaire, est la source d'inspiration type $\mathrm{du}$ texte de Barthes, une source plus ou moins explicite, puisque certaines mythologies portent directement sur des photos - les photos d'Harcourt, les " photos-chocs » ou encore celles de l'exposition La Grande Famille des hommes -, certaines portent sur des films, tandis que d'autres tendent à « dissoudre " la référence visuelle ${ }^{15}$. Le mythe, dit Barthes, « est un système sémio-

\footnotetext{
Miller, Paris, Seuil, 1978, pp. 270-27I.

I2 Je prends ainsi quelques distances avec l'idée de Susan Sontag selon laquelle Barthes aurait été un «écrivain heureux " à la « sensibilité post-tragique " (L'Écriture même. À propos de Barthes, trad. Philippe Blanchard, Paris, Christian Bourgois, 1982, pp. 37-38). La tragédie est passée, mais elle n'est pas loin.

I3 Voir en particulier, à propos de La Chambre claire, Jousset, « Morales du cliché », art. cit., et à propos des Fragments, Éric Marty, Roland Barthes, le métier d'écrire, Paris, Seuil, 2003, pp. 2102I4.

I4 Barthes, Mythologies, in Euvres complètes, op. cit., t. I, p. 823. Dorénavant, les références à cet essai seront indiquées par le sigle $M$ et placées entre parenthèses dans le texte.

I5 Jacqueline Guittard, «Impressions photographiques : les Mythologies de Roland Barthes », Littérature, I43, 2006, pp. II4-34. Pour l'édition illustrée : Roland Barthes, Mythologies, éd. Jacqueline Guittard, Paris, Seuil, 2010.
} 
logique second» (M, 828). D'un signe, comportant un signifiant et un signifié, d'après la linguistique saussurienne, il fait un signifiant auquel il attribue un autre signifié, mythique. Barthes en donne deux exemples. Le premier est emprunté à Paul Valéry : il s'agit de la citation d'une fable de Phèdre, quia ego nominor leo, trouvé dans une grammaire latine, où elle ne signifie plus ce qu'elle signifie, à savoir : car moi je mappelle lion, mais qu'elle est « un exemple de grammaire destiné à illustrer la règle d'accord de l'attribut » $(\mathrm{M}, 829)$. Il y a donc un premier signe, linguistique en l'occurrence, et ce signe se voit réduit à un signifiant inscrit dans un système sémiologique second, le système de la grammaire latine, où le patrimoine littéraire se trouve mobilisé afin d'exemplifier la règle grammaticale.

Mais cet exemple est insuffisant à illustrer le mythe, parce que peu marqué idéologiquement, et aussi parce qu'il n'est pas visuel, si bien que c'est davantage l'autre exemple que l'on retient justement :

Je suis chez le coiffeur, on me tend un numéro de Paris-Match. Sur la couverture, un jeune nègre vêtu d'un uniforme français fait le salut militaire, les yeux levés, fixés sans doute sur un pli du drapeau tricolore. Cela, c'est le sens de l'image. Mais, naïf ou pas, je vois bien ce qu'elle me signifie : que la France est un grand Empire, que tous ses fils, sans distinction de couleur, servent fidèlement sous son drapeau, et qu'il n'est de meilleure réponse aux détracteurs d'un colonialisme prétendu, que le zèle de ce noir à servir ses prétendus oppresseurs ( $\mathrm{M}, 830)$.

Tandis que l'autre exemple est emprunté à Valéry, celui-ci est personnel et mis en scène. Il préfigure l'avènement du Spectator, de ce sujet spectateur dont Barthes fera, dans La Chambre claire, la mesure du savoir photographique. Le sujet est ici inscrit dans une situation - «chez le coiffeur »-qui dit sa relative distraction, d'autant mieux qu'il n'est pas même à l'origine de l'apparition de ce numéro de Paris-Match, qu'« on [lui] tend ». La sollicitation mythologique vient d'ailleurs. Elle vient du monde, des circonstances de la vie quotidienne, à cette époque où les médias y acquièrent une place grandissante. Il s'agit ici d'une image en couverture, qui a un «sens» tenant dans sa description, sens qui se voit réduit et chapeauté par une signification mythique, idéologique et pro-colonialiste. Et cette signification, ajoute Barthes, est perceptible par tout un chacun. Elle est, autrement dit, stéréotypée. "Il y a enfin », dit encore Barthes, " une présence du signifié à travers le signifiant » (M, 830), phrase mystérieuse qu'il va s'appliquer à expliciter, et c'est cette explicitation qui m'intéresse ici plus particulièrement. Le signifiant du mythe a un double statut. Il est signe, autrement dit terme du premier système sémiologique, et signifiant, autrement dit base du second système sémiologique. Il est, dit Barthes, à la fois sens et forme, plénitude et surface : « dans le sens, une signification est déjà construite, qui pourrait fort bien se 
suffire à elle-même, si le mythe ne la saisissait et n'en faisait tout d'un coup une forme vide, parasite » $(\mathrm{M}, 83 \mathrm{I})$. Du sens à la forme, il y a " parasitage ", donc appauvrissement mais aussi vampirisme ou zombisme : "On croit que le sens va mourir, mais c'est une mort en sursis : le sens perd sa valeur, mais garde la vie, dont la forme du mythe va se nourrir » (M, 83I). Et Barthes, donc, de reformuler plus clairement ce qu'il entend par «présence du signifié » :

Le nègre qui salue n'est pas le symbole de l'Empire français, il a trop de présence pour cela, il se donne pour une image riche, vécue, spontanée, innocente, indiscutable. Mais en même temps cette présence est soumise, éloignée, rendue comme transparente, elle se recule un peu, se fait complice d'un concept qui lui vient tout armé, l'impérialité française (M, 832).

Il y a donc une présence du nègre représenté dans l'image, et cette présence est à la fois physique et intelligible. C'est à la fois un corps et un sens. Et ce corps signifiant vient naturaliser le mythe, faire du mythe de l'impérialité française une évidence aussi claire qu'une photographie.

\section{Présence de Michelet}

Si n'importe quel lecteur de Paris-Match peut lire un mythe, il faut un autre regard pour en faire la critique mythologique, ce que Barthes illustre en recourant là encore, significativement, au sens de la vue. Depuis le siège d'une automobile, dit-il, je puis accommoder soit sur le paysage soit sur la vitre. Dans le second cas, j'adopte le regard du mythologue. Le mythe m'apparaît comme une chose et sa signification s'en trouve distanciée :

L'impérialité française condamne le nègre qui salue à n'être qu'un signifiant instrumental, le nègre m'interpelle au nom de l'impérialité française ; mais au même moment, le salut du nègre s'épaissit, il se vitrifie, il se fige en un considérant éternel destiné à fonder l'impérialité française. À la surface du langage, quelque chose ne bouge plus : l'usage de la signification est là, tapi derrière le fait, lui communiquant une allure notificatrice ; mais en même temps, le fait paralyse l'intention, lui donne comme un malaise d'immobilité $(\mathrm{M}, 838)$.

Parce qu'il s'arrête sur l'image, parce qu'il chosifie le mythe, le mythologue n'en est pas dupe. Il n'y voit pas une nature, mais un être hors-nature, vampirisé, zombifié. «Le mythe, dit Barthes, est [...] un langage qui ne veut pas mourir : il arrache aux sens dont il s'alimente une survie insidieuse, dégradée, il provoque en eux un sursis artificiel dans lequel il s'installe à l'aise, 
il en fait des cadavres parlants » (M, 845). Le mythe, c'est déjà M. Valdemar. Mais ce sont d'abord, si l'on remonte aux sources des préoccupations littéraires et intellectuelles de Barthes, les corps de l'Histoire tels que Jules Michelet, le grand historien romantique, s'y est affronté. Barthes publie son Michelet en 1954, dans le sillage du Degré zéro de l'écriture, paru en 1953, et trois ans avant les Mythologies, et il s'agit en fait de son premier livre si l'on considère que Barthes commence à y travailler une décennie plus tôt, alors qu'il soigne sa tuberculose au sanatorium de Saint-Hilaire-du-Touvet. C'est sur Michelet qu'il publie son premier grand texte, en I95I, dans la revue Esprit, un article repris dans la monographie sous un titre qui dit frontalement une différence des corps, chez l'historien, que Barthes ne cessera jamais de retrouver dans son propre rapport aux corps et aux formes : celui entre « mort-sommeil » et « mort-soleil ».

L'Histoire micheletienne, rappelle Barthes, se veut une résurrection des corps du passé. Mais tous les corps ne se prêtent pas à cette opération qui révèle « le style d'une existence. Certaines sont des fausses morts, des morts apparentes, des demi-morts, ni mort ni vie $»^{16}$, ces morts-sommeil auxquelles s'opposent donc les morts-soleil. Barthes rapporte que Michelet a souhaité lui-même " qu'à sa mort son corps [soit] exposé au soleil jusqu'à dissolution ", mais que, cruelle ironie de l'Histoire, sa veuve « a préféré lui donner un caveau officiel et tarabiscoté au Père-Lachaise. Jules Ferry y prononça un discours oublié : l'ensommeillement radical-socialiste commençait et Michelet entrait dans cet enchantement immobile dont il avait toujours eu si grande peur » (MI, 293). Dans cet essai qui ne regarde pas, comme les Mythologies, du côté de Marx et de la critique de l'idéologie, mais du côté de Bachelard et de la critique thématique, dans cette " pré-critique » (MI, 353) de Michelet, comme Barthes la définit, la mort-sommeil est donc déjà identifiée au mythe, au " parasitage » institutionnel radical-socialiste dont sont victimes l'historien et son œuvre. Barthes note que les sommeils de l'Histoire donnent naissance à deux figures monstrueuses aux yeux de Michelet, le copiste et l'honnête homme :

L'Histoire endormie produit pire que le néant : le radotage. Le Moyen Âge devient vite une civilisation de copistes : dès I30o, l'architecture ne fait que se répéter à l'infini : plus de chants, plus de livres. Les églises se volent les unes les autres : des marbres de Ravenne, on fait Aix-la-Chapelle ; la société entière reste prisonnière du premier et du dernier mot du Moyen Âge : l'Imitation. Même aplatissement aux siècles monarchiques; le copiste médiéval est devenu ici «l'honnête homme », personnage stérile, calfeutré et ergotant, tel Montaigne, faible et négatif, tel le petit seigneur formé par les éducateurs

I6 Barthes, Michelet, in Euvres complètes, op. cit., t. I, p. 352. Dorénavant, les références à cet essai seront indiquées par le sigle MI et placées entre parenthèses dans le texte. 
classiques, Molière, Fénelon, Rousseau (MI, 329).

Autant de « cadavres parlants », de stéréotypes ambulants, donc, apparaissant dans les temps morts de l'Histoire.

Parmi ces figures ensommeillées del'Histoire micheletienne, il en est une qui mérite que l'on s'y attarde plus particulièrement, parce qu'elle fait signe pourtant vers une possible résurrection. Il s'agit de Napoléon Bonaparte, qui est inévitablement au centre de la dernière œuvre de Michelet, l'Histoire $d u X I x^{e}$ siècle, qui incarne pour lui l'ensommeillement postrévolutionnaire ; figure à la fois tyrannique et médiocre, puisqu'elle ne doit son succès, aux yeux de l'historien, comme le dit Barthes, qu'à la «Buona-Parte » (MI, 330) contenue dans son nom, au hasard ou à la grâce. L'interprétation micheletienne du personnage repose symboliquement sur son nom. Mais elle repose aussi sur l'image, plus précisément sur deux tableaux. D’une part, le portrait de Jacques-Louis David. Barthes cite ce passage de Michelet où celui-ci, décrivant ce portrait en pied, y voit une figure fantasmagorique, rabougrie, rongée de l'intérieur, autrement dit un mort-sommeil. Mais il est une autre représentation de Napoléon, célèbre, qui retient l'attention de l'historien comme celle de Barthes. Il s'agit de La Bataille d'Eylau d'Antoine-Jean Gros où l'empereur, blême, sur son cheval, fait un signe de la main dans lequel Michelet voit déjà un instant unique d'humanité et de compassion face à l'horreur de la guerre ${ }^{\mathrm{I} 7}$ et dans lequel Barthes voit plus fondamentalement " un numen » qui «ébranle le Temps " ${ }^{18}$ : "Si abîmé que soit le Napoléon de Michelet, il est de la même matière que celui de Gros, par exemple : tous deux ont cet empâtement irréel de l'homme héroïsé ; leur immobilité devant l'historien ou devant le peintre tient prisonnier un gestuaire, un style d'action : l'Histoire est fondée » $(\mathrm{MI}, 358)$. Il est significatif que, dans les Mythologies, le tableau de Gros revient comme exemple d'image réelle, s'opposant à la « photo-choc » qui elle ne touche pas parce que mythique, parce que stéréotype visuel (M, 75I-753). Il y a donc une solarité de Napoléon, et cette solarité apparaît dans le tableau de Gros. Par analogie, on peut dire qu'il y a du réel sous le mythe : c'est ce que Barthes explique à la fin des Mythologies, par exemple à propos du vin : " le vin est objectivement bon, et en même temps, la bonté du vin est un mythe » $(\mathrm{M}, 868)$. Il y a un réel inaliénable, mais de ce réel le mythologue ne peut parler, regrette-t-il, sinon de manière détournée, et c'est en gardant à l'esprit cette dernière considération de Barthes que j'aimerais me pencher plus longuement pour conclure sur la première mythologie, «Le Monde où l'on catche » (1952).

I7 Jules Michelet, Histoire du XIX siècle, in Euvres complètes, t. XXI, éd. Paul Viallaneix, Paris, Flammarion, 1982, p. 575.

I8 Barthes, « Michelet, l'Histoire et la Mort », in Euvres complètes, op. cit., t. I, p. II9. 


\section{Le Christ catcheur}

Cette mythologie est accompagnée exceptionnellement d'une épigraphe, une citation de Baudelaire qui revient régulièrement sous la plume de Barthes : «La vérité emphatique du geste dans les grandes circonstances de la vie» (M, 679). Marielle Macé s'est focalisée sur cette citation récurrente, dans son essai sur la lecture littéraire comme individuation, afin de pointer chez Barthes une préoccupation pour la stylisation de l'existence ${ }^{19}$. Pour ma part, j'aimerais seulement rappeler ce dont parle explicitement Baudelaire dans cette citation. Ce qu'il désigne, en fait, ce sont les figures de suppliants dans les tableaux de Delacroix. L'écrivain compare deux tableaux de Delacroix, La Justice de Trajan et l'Entrée des Croisés à Constantinople. Dans le premier, une "veuve éplorée $~^{20}$, dit Baudelaire, s'incline devant l'empereur, les bras écartés, afin de lui demander justice pour la mort de son fils. Dans le second, un vieil aveugle agenouillé, soutenu par sa femme, étend son bras en direction des Croisés, comme pour implorer leur clémence. Baudelaire insiste, dans une énumération, sur la proximité des deux représentations, en terminant sur ces figures centrales : "Et toujours ces drapeaux miroitants, ondoyants, faisant se dérouler et claquer leurs plis lumineux dans l'atmosphère transparente ! Toujours la foule agissante, inquiète, le tumulte des armes, la pompe des vêtements, la vérité emphatique du geste dans les grandes circonstances de la vie $! »^{21}$. Ce geste accentué, stylisé, et en même temps emphatiquement vrai, c'est donc manifestement un geste qui en appelle à la pitié. Et on sait que cet affect, la pitié, a une place centrale dans l'œuvre de Barthes, en particulier dans La Chambre claire ${ }^{22}$ (mais déjà au début des années 50, le critique se montrait sensible à la " charité » cayrolienne $)^{23}$. Barthes compare le catch à la tragédie (il « participe à la nature des grands spectacles solaires », M, 679), et ce rapprochement n'est nullement ironique : il est proposé encore et très sérieusement dans un article pour le Théâtre populaire à la même époque $(1953)^{24}$. Le catch est comparé à la tragédie, et opposé à la boxe comme à une autre forme de récit :

Le match de boxe est une histoire qui se construit sous les yeux du spectateur ; au catch, bien au contraire, c'est chaque moment qui est intelligible, non la durée. [...] Chaque moment impose la connaissance totale d'une passion qui surgit droite et seule, sans s'étendre jamais vers le couronnement

I9 Marielle Macé, Façons de lire, manières d'être, Paris, Gallimard, 20II, pp. I82-268.

20 Charles Baudelaire, «Exposition universelle (I855) ", in Critique d'art suivi de Critique musicale, éd. Claude Pichois, Paris, Gallimard, 1992, p. 252.

2I Ibid.

22 Barthes, La Chambre claire, in Euvres complètes, op. cit., t. V, pp. 880-883.

23 Id., "Jean Cayrol et ses romans », in Euvres complètes, op. cit., t. I, pp. I49-I54.

$24 I d$., « Pouvoirs de la tragédie antique ", ibid., pp. 259-267. 
d'une issue $(M, 680)$.

La boxe est narrative au sens où elle crée chez le spectateur une tension que seul le dénouement pourra résoudre, tandis que la tragédie est, comme le théâtre de Diderot, une succession d'instants prégnants. Barthes a, depuis son premier article, «Culture et tragédie » $(1942)^{25}$, une conception dénarrativisée, détemporalisée du récit tragique : ébranlement du Temps, manifestation d'un hors-Temps numineux. Le catch, comme la tragédie, est une succession de tableaux.

Dans ces tableaux vivants, il y a une évidence stéréotypée des gestes : « un homme à terre y est exagérément, emplissant jusqu'au bout la vue des spectateurs, du spectacle intolérable de son impuissance » (M, 680). Il y a aussi une évidence stéréotypée des rôles : en l'occurrence, celui de Thauvin, « quinquagénaire obèse et croulant » qui incarne le « salaud-pieuvre » (M, 680-68I) : ce n'est plus ici la tragédie, mais la commedia dell'arte que Barthes mobilise pour dire le respect, de la part du catcheur, d'un authentique canevas. "Le physique des catcheurs institue donc un signe de base qui contient en germe tout le combat » $(\mathrm{M}, 68 \mathrm{I})$, remarque-t-il, c'est-à-dire qu'il en va du corps du catcheur comme du Mot dans la poésie post-hugolienne (" sous chaque Mot de la poésie moderne gît une sorte de géologie existentielle, où se rassemble le contenu total du Nom $»^{26}$ ) comme du Nom dans l'Histoire micheletienne (Bonaparte, la Bonne Part) : il contient en germe un style existentiel. Mais ici le style ne renvoie pas à la substance irréductible de l'écrivain, mais à une substantification du type : il s'agit de donner corps, le plus littéralement possible, à un rôle. «Ce que le public réclame, c'est l'image de la passion, non la passion elle-même » $(\mathrm{M}, 682)$ : une formule qui revient, modifiée, à propos de la photo-choc : « la photographie littérale introduit au scandale de l'horreur, non à l'horreur elle-même » $(\mathrm{M}, 753)$. Dans le cas de la photo, il s'agit de dire que celle-ci n'est littéralement choquante qu'à la condition d'être naturelle, de ne pas «surconstrui[re] l'horreur » $(\mathrm{M}, 752)$. Ici, il s'agit de se conformer à l'emphase du numen pictural :

Le catch présente la douleur de l'homme avec toute l'amplification des masques tragiques : le catcheur qui souffre sous l'effet d'une prise réputée cruelle [...] offre la figure excessive de la Souffrance ; comme une Pietà primitive, il laisse regarder son visage exagérément déformé par une affliction intolérable $(\mathrm{M}, 683)$.

Le catch offre donc une image. Mais il s'agit d'une image picturale (ou

25 Id., « Culture et tragédie », ibid., pp. 29-32.

26 Id., Le Degré zéro de l'écriture, in Euvres complètes, ibid., p. 199. 
sculpturale) et non photographique, d'un artefact et non de la réalité brute, malgré la présence réelle des corps sur le ring. Dans son article pour le Théâtre populaire, Barthes dit encore qu'« au catch, la perfection de la mimique rend inutile l'authenticité du combat » comme " au théâtre, l'extériorité des signes rend dérisoire le réalisme » : «Il y a là une manière d'intensité qui charge le combat sportif ou le spectacle tragique d'une véritable jubilation de l'intelligence, parvenue à une saisie immédiate des rapports, et non des choses, ce qui est la définition même de la culture $»^{27}$. La vertu du catch, spectacle littéral et néanmoins culturel, c'est qu'il renoue avec le degré zéro de l'écriture tragique.

Pictural, le catch n'en demeure pas moins, en effet, un théâtre, c'est-àdire que le corps du catcheur y est signifiant tout en imposant sa présence : " la mollesse des grands corps blancs qui s'écroulent à terre d'une pièce ou s'effondrent dans les cordes en battant des bras " (M, 684). Dans l'article qu'il consacre en 1954 au "Théâtre de Baudelaire ", Barthes pointe la duplicité du corps de l'acteur baudelairien, «à la fois corps vivant venu d'une nature triviale, et corps emphatique, solennel, glacé par sa fonction d'objet artificiel $\aleph^{28}$. De la même manière, le catcheur est un corps vivant glacé par sa fonction mythique, ce mythe étant une parole elle-même pure de tout parasitage : « ne laissant rien dans l'ombre, le geste coupe tous les sens parasites et présente cérémonialement au public une signification pure et pleine, ronde à la façon d'une Nature » $(\mathrm{M}, 687)$. Elle-même pure, elle ne parasite pas le corps, mais s'y ajoute, l'augmente et le ressuscite :

Au catch, [...] la Défaite [...] est une exposition, elle reprend les anciens mythes de la Souffrance et de l'Humiliation publiques : la croix et le pilori. Le catcheur est comme crucifié en pleine lumière, aux yeux de tous. J'ai entendu dire d'un catcheur étendu à terre : "Il est mort, le petit Jésus, là, en croix ", et cette parole ironique découvrait les racines profondes d'un spectacle qui accomplit les gestes mêmes des plus anciennes purifications (M, 684).

Le catcheur est un Christ laïque, comme celui de Michelet. Il tient un rôle mythique, immédiatement reconnaissable, mais c'est un mythe "profond » et pitoyable, que l'ironie ou le sarcasme ne peuvent corrompre. " Il est mort et il va mourir ${ }^{29}$, comme le condamné à mort de la photo d'Alexander Gardner, dans La Chambre claire. "Crucifié en pleine lumière ", il est mort-soleil, appelé en tant que tel à la résurrection. Au reste, si le catch, comme avatar moderne de la tragédie, est transfiguration de la réalité, on

27 Id., « Pouvoirs de la tragédie antique », art. cit., pp. 263-264.

28 Id., "Le Théâtre de Baudelaire ", in Euvres complètes, op. cit., t. II, p. 306.

29 Id., La Chambre claire, op. cit., p. 867. 
peut dire non moins justement que la tragédie est ce mythe « profond » qui vient transfigurer, dans l'écriture, la stéréotypie du catch. Sous la plume de Barthes, le catch n'est plus tout à fait - voire plus du tout - du catch et les catcheurs ne sont plus tout à fait - voire plus du tout - des catcheurs. Ils sont à la fois plus réels et plus irréels, plus physiques et plus métaphysiques. En ce sens également, il y a résurrection. 